Mots. Les langages du politique

\title{
Le cyclone Mitch et le catastrophisme du Monde
}

\section{Dominique Teissier}

\section{CpenEdition}

\section{Journals}

Édition électronique

URL : https://journals.openedition.org/mots/3433

DOI : $10.4000 /$ mots.3433

ISSN : 1960-6001

\section{Éditeur}

ENS Éditions

\section{Édition imprimée}

Date de publication : 1 juillet 2004

Pagination : 101-110

ISBN : 2-84788-057-7

ISSN : 0243-6450

\section{Référence électronique}

Dominique Teissier, «Le cyclone Mitch et le catastrophisme du Monde », Mots. Les langages du politique [En ligne], 75 | 2004, mis en ligne le 22 avril 2008, consulté le 23 avril 2022. URL : http:// journals.openedition.org/mots/3433; DOI : https://doi.org/10.4000/mots.3433 
Dominique TEISSIER ${ }^{1}$

\section{Le cyclone Mitch et le catastrophisme du Monde}

La violence des faits climatiques qui agitent la planète nous rappelle régulièrement l'impuissance humaine. Les médias d'information construisent ces faits remarquables en évènements. Nous nous sommes intéressée à cette stratégie discursive sous un aspect particulier, le traitement de l'émotion. Pour cela, nous avons choisi d'étudier le cas du cyclone Mitch, qui a atteint l'Amérique centrale en novembre 1998. Un cyclone est un objet à fort potentiel émotionnel: l'homme est placé face à un élément naturel qui génère la désintégration sociale. Dans le foisonnement des discours qui en ont rendu compte, notre corpus d'étude s'est limité à des articles issus du Monde des 16 et 17 novembre 1998: un titre de référence de la presse d'information pouvait-il résister à cette tendance qui donne à voir l'émotion de l'autre et invite à une communion en elle?

Notre perspective de travail n'est pas de savoir si la représentation de l'émotion par la presse suscite chez les lecteurs une réelle réaction émotive. Ce qui nous semble significatif est le recours à l'émotion, peut-être partagée par les lecteurs, mais assurément mise en scène par Le Monde. Une attention particulière se portera sur les trois journées des 6,8 et 10 novembre, suivant en cela la logique du journal, qui réservait à l'évènement une pleine page ou davantage. Les autres jours sont, eux aussi, intéressants mais ils servent plutôt de lien entre des temps forts du discours car le fil conducteur d'un tel drame ne peut être brutalement rompu. Analyser comment est prise en compte l'émotion dans Le Monde nous permettra d'avancer dans la compréhension d'une stratégie discursive tablant sur l'émotion, stratégie appelée «pathique».

\section{La stratégie pathique dans le discours du Monde}

Nous allons tenter de décrire l'émotion comme topique (parler d'elle) et démarche stratégique (la re-susciter pour convaincre). Lorsque Le Monde l'in-

1. IUT de Châteauroux - Université d'Orléans - dominique.teissier@univ-orleans.fr 
tègre, il ne s'agit pas, pour le journal, d'émouvoir pour émouvoir mais de reconstruire par le discours le partage symbolique, absent du temps pendant lequel on la vit. Un fait tel qu'un cyclone renvoie l'homme à une impuissance fondamentale: celle de la maitrise des éléments naturels. Face au danger, le corps exprime une tension difficilement contrôlable. Or le langage, en tant que moyen d'appréhension du réel, ne confère à l'individu que l'illusion d'un contrôle : l'homme maitrise sa création langagière, non le réel. La parole, parce qu'elle re-présente, est rassurante: le lecteur du quotidien sait que le danger n'est pas ici et maintenant. Mais, au moment de l'épreuve, le langage, médiation de la structuration sociale, est absent. Le temps de l'émotion est une expérience singulière que le sujet n'assume pas et ne peut partager. Elle est doublement paralysante, car elle remet en cause l'identité du sujet et l'organisation sociale à travers la suspension de la parole. Pour Patrick Charaudeau, l'information en tant que genre discursif se joue sur deux scènes : la scène des effets de réel, lieu fabriqué pour répondre à l'angoisse de la solitude «vue comme un isolement de sa propre expérience $»^{2}$, et la scène des effets de fiction, permettant d'avoir une vision totale de la (et de sa) destinée par la médiation de l'imaginaire.

Ressusciter l'émotion (de l'autre/de soi) et inviter à son partage symbolique permettrait de donner une valeur objective et consensuelle à cette expérience individuelle. Mettre en mots le temps vécu de l'émotion et de la souffrance peut permettre au sujet de renouer avec le social. L'émotion est alors mise en scène à deux niveaux d'énonciation:

- elle est représentée dans un discours assumé par un énonciateur; il peut s'agir des témoignages des victimes que le discours de presse organise (discours rapporté, direct ou indirect) ou du témoignage assumé par l'auteur de l'article;

- elle est supposée partagée par les énonciataires du discours.

Dans les deux cas, il s'agit de la construire comme un objet de savoir qui puisse devenir référence. Ce processus s'appuie sur deux procédés imbriqués :

- la fabrication d'une image de réel comme lieu d'une vérité extérieure au sujet;

- la fabrication d'une image de fiction comme lieu d'identification du sujet à un autre lui-même, et de projection de l'imaginaire de ce sujet ${ }^{3}$.

Cette stratégie vise à recréer de la cohésion dans le temps partagé de l'émotion, alors que ce temps même est suspension de l'appartenance sociale. Quel enjeu peut-elle révéler? Peut-être celui d'un renouveau du contrat de communication, selon lequel «éprouver de l'émotion, c'est apprendre ${ }^{4}$. Le discours

2. P. Charaudeau, 1983, p. 97.

3. P. Charaudeau, 1983, p. 95.

4. Selon l'adage grec pathein, mathein. 
du Monde a recours, dans un premier temps, à la figure de la catastrophe car on ne peut pas ne pas être ému (règle sociale). Cependant, la généralisation de la stratégie pathique dans les médias d'information présente un risque: celui de la dépolitisation de l'espace public. Car l'émotion n'est pas en débat, elle ne laisse pas place à la polémique: elle est consensus immédiat par la réactivité individuelle. On retrouve là l'une des stratégies du discours populiste. Tout dépend de la finalité de l'énonciateur dans le recours à la stratégie pathique et du statut de l'émotion mobilisée.

Dans le cas étudié, l'éditorial du 8 novembre nous éclaire. Le pathétique est l'occasion, dans un deuxième temps, d'une leçon morale et politique. Cette leçon permettra, dans un troisième temps, de mobiliser la solidarité internationale par l'appel à la compassion. La stratégie pathique, dans ces articles du Monde, est donc une construction discursive qui intègre l'émotion supposée de l'autre sur la scène des effets de réel, qui invite à un partage de la souffrance sur la scène des effets de fiction et qui donne lieu à une leçon de solidarité.

\section{La catastrophe dans la topique de l'émotion}

Le 6 novembre, la une du journal annonce: «Le Honduras et le Nicaragua dévastés par le terrible ouragan Mitch». La page 2 s'ouvre par le mot catastrophe dans le surtitre. Ce mot construit un horizon d'attente émotionnel. Le discours du Monde va œuvrer, sur une pleine page, à la construction du sens de cette catastrophe. Trois thématiques se dégagent des articles:

- la catastrophe naturelle (champ lexical des éléments déchainés: ouragan, cyclone, glissement de terrain, inondations, pluies torrentielles, coulées gigantesques, tremblement de terre, torrents de boue). Les termes sont porteurs de référence à l'émotion publique; ils renvoient à une terreur fondamentale;

- la catastrophe humanitaire: comptabilité morbide à l'heure du bilan «provisoire» qui «s'alourdit d'heure en heure»: «quelque 10000 morts, près de 14000 disparus et 2,8 millions de sinistrés » (indication mentionnée à trois reprises). La désignation de l'humain renvoie à la mort et à la souffrance (morts, disparus, sinistrés, victimes, piétons chargés comme des mules, retour à l'animalité, survivants, blessés), ainsi qu'à la construction d'une scène d'horreur (cadavres des victimes, cadavres en décomposition, corps déchiquetés);

- la catastrophe économique s'évalue en «milliards de dollars» et comporte la «destruction des routes, des ponts, de toutes les infrastructures», etc. La perte du lien social engendre l'émotion en raison de l'isolement, de la peur, de l'impossibilité de porter secours ou de fuir (les habitants «cherchent désespérément un moyen de transport pour fuir les lieux»). Ces catégories se combinent pour 
produire par concaténation le sens général de la catastrophe. L'aboutissement se retrouve dans le champ lexical de la destruction (détruire, ravager, dévaster, périr, mourir, entrainer la mort, tout emporter...).

La volonté de donner au désastre toute sa portée par des évaluations démesurées, de faire imaginer l'inimaginable, de dire ce qui ne peut l'être, est plus significative encore. La difficulté consiste à décrire de façon crédible une réalité qui touche à l'incroyable. On évoque pour cela «l'amas indescriptible» formé par «des restes d'arbres, des pierres, des cadavres d'animaux gonflés d'eau et même une énorme pelleteuse ». Dans une longue description de villages et de sites détruits, le surgissement du discours direct établit une rupture:

"On a repéré huit nouveaux corps», annonce un petit paysan trapu au lieutenant Dionisio Narvaez, qui fournit aussitôt l'essence nécessaire pour incinérer les cadavres [...] pour limiter les risques d'épidémies. (Le Monde, 6 novembre 1998)

La catastrophe, c'est le dénouement d'une tragédie. Le discours de presse emprunte au tragique à travers le pathétique du miracle, dans un univers de mort (une femme retrouvée, son bébé dans les bras, dans un torrent de boue, roulée à plus de cent kilomètres à l'heure parmi les corps déchiquetés), et d'impuissance: «La nuit, [...] on entend les gémissements des survivants mais on ne dispose pas des moyens nécessaires pour les sortir de là». Le commentaire ouvre sur l'imaginaire, sur l'incertitude et l'inéluctable: «Combien de cadavres resteront ensevelis dans cette mer qui s'étend sur plus de [...]. On ne le saura sans doute jamais...».

Parallèlement, l'article «Un des ouragans les plus terribles du siècle» tente de rationaliser l'effet du cyclone par la compréhension du phénomène physique. Un graphique décompose les trois temps de sa formation. Le récit va permettre la contextualisation spatio-temporelle du fait ${ }^{5}$, rendu objectif par focalisation externe. Mais cette focalisation est rompue dès qu'une trace d'énonciation apparait: «Les pluies diluviennes ont ainsi matraqué une région circonscrite, provoquant des inondations d'autant plus destructrices que...». L'italique signale ici une intervention directe dans le récit objectivant, soit que le terme n'ait rien à voir avec les références scientifiques qui l'accompagnent (niveau de langue), soit qu'il vise à orienter la lecture vers la violence du phénomène. La rationalisation tend à détacher le fait de son contenu émotionnel, mais le titre ainsi que la mise en scène polyphonique concourent à entretenir la peur devant cette violence. Et le journal de poser la question: «L'intensité des cyclones et leur fréquence découleraient-elles du réchauffement climatique qui s'amorce?».

5. Cette contextualisation s'opère également dans l'énumération chronologique des principaux glissements de terrain (texte AFP) depuis 1985, sur le principe : datation du fait, localisation, nombre de victimes. La nouvelle catastrophe entre dans l'histoire et deviendra référence. 
La réponse des scientifiques, par sa prudence et l'impuissance qu'elle révèle, est loin de rassurer («Certes [...], en principe [...], mais il faudrait pouvoir [...], ce que l'on ne sait pas encore faire.»): la tentative de rationalisation est pour l'instant tenue en échec, et le lecteur invité à accepter la fatalité. Cependant, la légende du graphique ( «Un phénomène climatique propre aux zones tropicales chaudes»), en localisant le phénomène chez les autres, peut à la fois éloigner le danger et amorcer la compassion ultérieure du lecteur.

Mitch, en fin de compte, est une non-personne, élément tragique constituant la menace meurtrière (action maléfique irrationnelle), qui est devenue réalité et se termine en catastrophe. La représentation du fait tragique relève d'une approche pathique. L'évènement passe par la construction spectaculaire du fait de nature à émouvoir. Il est largement fait appel à la perception du sujet dans sa mise en scène, et le lecteur se trouve renvoyé non à un procès de signification mais à un pathos plus ou moins inscrit dans l'imaginaire individuel et collectif. Le lien entre perception et pathos est établi par la figure du tangible, «ce contact prétendu direct avec le monde qui engendre le mythe du témoin-spectateur $\rangle^{6}$. Le discours de presse construit ainsi des effets qui permettent au lecteur de vérifier le réel comme s'il en était spectateur, de partager l'horreur de la catastrophe figée dans l'illusion du direct.

Le 7 novembre, s'amorce le thème de la catastrophe en chaine et les derniers bilans sont présentés. "Après Mitch, la famine», titre la une; «les épidémies [...], la faim et la maladie sont les nouveaux ennemis à combattre». En page 38, les derniers comptes macabres sont rapportés. On retrouve les mêmes champs lexicaux et les descriptions répugnantes où les sens sont sollicités (décomposition, puanteur, insalubrité). Le réalisme domine, non en ce qu'il s'oppose à l'imaginaire, mais en ce qu'il lui sert de tremplin par les références culturelles qu'il mobilise. À travers le thème de la mort, l'émotion est liée à l'impossible respect des rituels et du travail du deuil:

Les morts sont souvent abandonnés sur place aux vautours et aux chiens, trop hâtivement enterrés. Abandonnés parmi les décombres, les corps sont la proie des vautours, notamment sur les berges nauséabondes... Les sinistrés [isolés] guettent le ciel dans l'espoir qu'un hélicoptère...

\section{«Mitch a surement beaucoup ému»}

Jusque-là, la localisation spatiale de la catastrophe tendait à dissocier les deux pays concernés («Au Nicaragua», «Au Honduras»). À partir du moment où

6. P. Charaudeau, 1983, p. 98. 
l'aide internationale commence à s'organiser, une aire spatiale nouvelle se profile: la «zone dévastée par le cyclone». La première carte qui la représente apparait le 8 novembre. Elle dessine un périmètre au sein duquel les frontières sont effacées. Une légende localise les catastrophes naturelles, une autre hiérarchise par pays le bilan de la catastrophe humanitaire. Celle-ci transcende les frontières, et les enjeux politiques s'effacent devant la nécessité et la contrainte morale. Mais les politiques se livrent à une guerre des chiffres, espérant «attirer l'aide étrangère sur leurs territoires respectifs». Témoins, les propos du ministre de l'Intérieur du Honduras: "Il n'est pas nécessaire de dédramatiser ni d'exagérer l'ampleur du désastre. Les faits parlent d'eux-mêmes. C'est la catastrophe la plus grave de notre histoire et la communauté internationale l'a bien compris...». Mais si les faits parlent d'eux-mêmes pour les victimes du cyclone, les médias parlent des faits pour les autres. La représentation de la catastrophe, construite par le discours d'information, a produit le savoir nécessaire à la légitimation de l'action politique.

Pour autant, le pathos ne disparait pas du discours tenu en deuxième page. Nous retrouvons les descriptions réalistes et de nouvelles ruptures énonciatives qui suscitent l'illusion référentielle:

« J'ai perdu mon travail et mon patron n'a plus d'entreprise. Tout a été détruit par l'eau et la boue. Ç'aurait pu être pire», soupire-t-il, en regardant le ballet terrifiant des vautours qui picorent les cadavres d'animaux en plein cœur de la capitale. Ce spectacle hallucinant se déroule au pied de l'ancien palais présidentiel, récemment converti en musée, et à une centaine de mètres du Parlement [...]. La scène se répète dans d'autres points de la capitale... (Le Monde, 8 novembre 1998)

En page 2 encore, cette dépêche AFP: «J'ai vu mon fils avalé par une vague... » Il s'agit du témoignage d'une «jeune femme, Isabella Arriola, professeur de 36 ans [qui] pleure la disparition de ses trois enfants et de son mari». Représentation de l'émotion de l'autre, mais aussi invitation à l'empathie dès le titre. Celui-ci est un fragment de discours direct qui, ainsi placé, acquiert un statut autonome. Par contre, un glissement de la stratégie pathique (dont le texte porte des traces) à la stratégie éthique est effectué dans l'article «Du cyclone à la schizophrénie planétaire». Il s'agit maintenant de signifier ce que l'énonciateur veut être pour l'autre, de «délibérer sur le fait, afficher une franchise qui ne craint pas ses conséquences, s'exprimer à l'aide de propos directs, empreints d'une loyauté théâtrale, entrer dans une complicité complaisante à l'égard de l'auditoire» ${ }^{7}$.

Car la catastrophe aboutit à une leçon morale et politique sur les thèmes de l'équité et de l'intelligence: «Rien ne peut se faire si les nations riches ne sont pas convaincues que leur devoir est d'aider les plus pauvres à faire face au

7. R. Barthes, 1970. 
péril commun». Quant à la complaisance, elle se présente sous l'aspect de l'indulgence vis-à-vis de soi et des autres, à travers le pronom on réunissant énonciateur et énonciataire: «on le voit», «que l'on ne sache pas bien mesurer la valeur économique de la diversité n'empêche pas que...», «on peut trouver le moyen de...». Il s'agit de passer du savoir au comprendre pour agir.

\section{De la compassion à la mobilisation}

Le 10 novembre, un nouveau dispositif met en scène l'émotion dans trois sites différents: la une, un reportage en page 4, et deux longs témoignages en pages 14 et 15 . Pour la première fois dans la série tragique, l'image photographique est employée. De la vignette de la une (un homme surnage, à califourchon sur un morceau de bois) aux cinq photographies accompagnant les témoignages, le dispositif permet l'inscription d'un présent figé. En effet, la force constative de la photographie porte non sur l'objet mais sur le temps ${ }^{8}$. L'action photographiée est passée, l'image opère une suspension temporelle. Le premier témoignage ouvre sur le même temps mis en scène: «Je suis ici, assise face à mon ordinateur». Texte et images se font écho :

Le soleil fait des efforts pour sortir et il semblerait que tout est normal. Mais il suffit d'allumer la télévision, d'ouvrir les pages des journaux ou de se connecter sur Internet pour se rendre compte qu'il n'y a rien de normal. (Le Monde, 10 novembre 1998)

Les images de la tragédie que propose le journal témoignent de ce «rien de normal». L'anormalité dans l'image nait de la rupture entre l'horizon d'attente du titre et la représentation: «Mitch, l'ouragan catastrophe» renvoie aux éléments déchainés; la photo placée au-dessus du titre sur une demi-page représente le calme après la tempête, mais aussi la destruction. Car, si on ne peut rationaliser la figure cyclonique (fatalité), on peut agir sur ses effets destructeurs (solidarité internationale). Sur cette vue aérienne d'un village du Honduras inondé, le contraste vient de la composition: platitude suggérée par l'eau calme (silence?) qui nivelle l'espace jusqu'à la ligne d'horizon. Les seules traces de relief qui se détachent dans ce désert aquatique sont les maisons, arbres et buttes inondés. L'eau fige le paysage sur un miroir plan d'où l'humain est absent: le village est déserté et les signes paysagers du lien social, par exemple les routes, n'existent plus.

Sous le titre principal, un nouveau graphique représente la zone dévastée et le journal annonce: «des témoignages poignants où perce une vive critique des

8. R. Barthes, 1980. 
autorités de Managua et un appel à l'entraide». Suivent deux témoignages d'écrivains. Le premier s'intitule «La tragédie de Sisyphe». Les références mythiques nourrissent analogies et métaphores:

Le scénario est totalement apocalyptique, Dante, dans son Enfer, serait bien audessous du compte. [...] Tout cela ressemble à Pompéi. [...] Ici, on dit que le Nicaragua est le pays de Sisyphe. (Le Monde, 10 novembre 1998)

Le second témoignage ( «Il nous reste l'espoir») s'ouvre par la phrase «Une fois encore, nous revenons des décennies en arrière...». Espoir et solidarité de la population face à la catastrophe sont aussi des représentations communes. Le pathétique emprunte à la technique de la mimesis, en s'appuyant sur des scènes exemplaires :

À la Croix-Rouge, des gens très pauvres font d'immenses queues, afin d'offrir le peu qu'ils possèdent. Une femme, déjà vieille, me disait qu'elle possédait trois draps et qu'elle en avait donné deux. (Ibid.)

Les récits de comportements admirables invitent à la modélisation et à la réponse imitative. Car le partage de l'émotion peut s'effectuer sur deux modes: la symétrie ou la complémentarité. Face à l'horreur, au désespoir des victimes du cyclone, on peut à son tour être horrifié et désespéré, ou bien adopter une attitude de compassion. Ce comportement est placé très haut sur l'échelle de la moralité, et Le Monde nous invite à l'adopter. Il s'agit de dépasser la condition tragique par l'empathie et l'espoir en la reconstruction: «Cet esprit de piété, de compassion et de solidarité, aucune rancune ni confrontation n'a jamais réussi à le détruire. Et, aujourd'hui, nous l'attendons aussi du reste du monde.»

Les choix photographiques ont le même effet, qu'il s'agisse de personnes tentant de récupérer dans un courant de boue des biens dérisoires, d'un secouriste portant un enfant nu (mort ou endormi) ou d'une scène d'enterrement. Le photographe est dans le cortège, le point de vue à hauteur d'adulte est fixé sur un enfant portant un cercueil d'enfant plus jeune encore, et l'on perçoit comme une invitation à l'alléger de son fardeau. La mort d'un enfant ou sa souffrance sont des figures communes de la stratégie pathique:

Les enfants sont ceux qui m'impressionnent le plus. Il y en a encore qui sourient mais dans leur regard se reflète un je ne sais quoi de tristesse ou de sagesse énorme. Ils ont pris conscience de la mort...

... dans cette peinture ocre et grise apparaissent une main qui émerge, une jambe entre les branches, un enfant jeté dans la boue comme un jouet cassé. C'est l'image la plus éloquente de notre malheur. (Le Monde, 10 novembre 1998)

Admiration, compassion, espoir, autant de sentiments qui annoncent l'ardeur de la reconstruction. Cette exaltation sera encore plus lisible le 12 novembre. Il 
s'agit des «étudiants mobilisés pour déblayer les rues de Tegucigalpa ». Même si l'ombre des vautours plane encore sur le texte et sur les zones détruites, l'enthousiasme prévaut: «Nous sommes la génération Mitch et nous avons tous des ampoules aux mains». La communauté brandit après le cyclone un nouveau signe identitaire et confirme son existence dans l'action. La clôture de cet article permet de reprendre le cours de l'histoire, comme si le temps du cyclone n'avait été qu'une suspension momentanée de la logique sociale. Le lien entre «nous» et «les autres», entre «aujourd'hui» et «hier», est à nouveau établi :

Ah, les Mexicains! Heureusement qu'ils sont là, s'exclame Salvador Majano, propriétaire d'une armurerie. [...] Ils ont fait un travail fantastique et je sais pas comment on va pouvoir les remercier. Je suis même content qu'ils nous aient battus au football! (Le Monde, 12 novembre 1998)

La fin heureuse permet la réintégration du politique, la réaffirmation de la victoire du social sur le fatum et l'inscription dans la mémoire d'une scène de pathos qui pourra être réactivée.

La stratégie pathique mise en œuvre est peut-être différente selon qu'on s'adresse à des lecteurs ayant vécu l'émotion (cas de la tempête de décembre 1999 en France) où lorsqu'on s'adresse à un public pour lui faire partager une émotion vécue par d'autres, sous d'autres latitudes. Dans la tempête française, la référence émotionnelle passe par le «trou noir» (parce que proche du lecteur?) ${ }^{9}$; pour le cyclone Mitch, elle utilise les ressources de l'imaginaire: il faut à la fois rendre crédible ce que nous n'avons pas vécu et le construire comme incroyable. Lincroyable est présenté comme un aspect du crédible. La description réaliste, en reconnaissant qu'elle ne peut tout dire, rend le fait crédible et le construit dans un univers d'attente émotionnel: on lit plus que ce qui est écrit, on dépasse le discours, on imagine l'indescriptible.

La stratégie du Monde vise à cadrer cet affect: il ne s'agit pas de se laisser aller à une émotion réputée basse mais à une émotion noble, la compassion dynamique. En outre, le discours ne se limite pas à cette invitation au partage de la souffrance: il vise un positionnement éthique et politique. Articulant stratégie éthique, stratégie logique et stratégie pathique, Le Monde ne sombre pas au cœur de la tragédie; il s'en inspire largement pour construire une catastrophe qui donne lieu à une leçon morale et politique.

9. C. Jamet, «La stratégie de l'évanouissement», dans ce numéro de Mots, voir supra. 


\section{Bibliographie}

BARTHES R., 1980, La chambre claire: note sur la photographie. Paris, Gallimard (Cahiers du cinéma).

— 1970, «L'ancienne rhétorique : aide-mémoire», Communications, $\mathrm{n}^{\circ} 16$.

BIET C., 1997, La tragédie, Paris, Armand Colin.

BRETON P., 1996, L'argumentation dans la communication, Paris, La Découverte.

CHARAUDEAU P., 1983, Langage et discours : éléments de sémiolinguistique, Paris, Hachette.

— 1997, Le discours d'information médiatique, Paris, Nathan.

JAMET C. et JEANNET A.-M., 1999, Les stratégies de l'information, Paris, L'Harmattan.

LAMIZET B., 1992, Les lieux de la communication, Liège, Mardaga.

MAINGUENEAU D., 1990, Pragmatique pour le discours littéraire, Paris, Bordas.

- 1991, L'analyse de discours : introduction aux lectures de l'archive, Paris, Hachette.

- 1991, L'énonciation en linguistique française, Paris, Hachette.

MOUILLAUD M. et TÉTU. J.-F., 1989, Le journal quotidien, Lyon, PUL.

— 1997, «Comprendre les émotions », Sciences Humaines, ${ }^{\circ} 68$, janvier.

RIME B., 2000, «Faut-il parler de ses émotions?», Sciences Humaines, n 104, avril. 